\title{
Itinéraires croisés
}

\section{Michèle Ducornet}

\section{(2) OpenEdition}

\section{Journals}

Édition électronique

URL : http://journals.openedition.org/span/1626

DOI : $10.4000 /$ span. 1626

ISSN : 2268-1558

\section{Éditeur}

École pratique des hautes études. Sciences humaines

\section{Édition imprimée}

Date de publication : 1 janvier 2014

Pagination : 25-32

ISBN : 9782909036434

ISSN : 0294-7080

\section{Référence électronique}

Michèle Ducornet, «Itinéraires croisés », Systèmes de pensée en Afrique noire [En ligne], 19 | 2014, mis en ligne le 04 février 2020, consulté le 11 février 2020. URL : http://journals.openedition.org/span/ 1626 ; DOI : 10.4000/span.1626 


\title{
Itinéraires croisés
}

\section{Michèle Ducornet}

Psychanalyste

\begin{abstract}
"Toute âme est une mélodie qu'il s'agit de renouer; et pour cela, sont la flûte ou la viole de chacun. Selon moi jaillit une condition vraie ou la possibilité, de s'exprimer non seulement, mais de se moduler, à son grél."
\end{abstract}

C'est sous les auspices de Stéphane Mallarmé, que Michel Cartry aimait tant, que j'ai souhaité nous situer aujourd'hui parce que ça le représente bien.

Il aimait Mallarmé, la poésie, la musique. Il y retrouvait avec jubilation ce qui se trame au travers de la langue, qui nous précède et nous excède, ce que nous ignorons et qui survient dans la langue. Il était passionné de l'insu qui surgit avec elle, "dans ses plis " disait-il.

Ce n'est pas étonnant qu'il ait très tôt rencontré la psychanalyse dans sa quête assidue de la compréhension de l'humain.

Certes, Sigmund Freud a conçu la psychanalyse comme outil de soin. Mais, pour réaliser ce projet il lui fallut construire pas à pas une théorie des mécanismes psychiques à l'œuvre. Bien sûr c'est une théorie liée à son origine au lieu et à la temporalité de sa fabrication et qui n'a cessé d'évoluer avec Freud lui-même et les psychanalystes qui lui ont succédé pour le meilleur et pour le pire. 
2 Extrait de la préface au premier volume de James George Frazer, (1910).

Jacques Lacan, 1954.
Pourtant quelque chose ne cède pas, c'est la coexistence dans la pensée de deux registres engrenés l'un dans l'autre. L'un est celui auquel nous avons directement accès par la conscience, l'autre constitué de traces, aussi bien phylogénétiques que généalogiques et relevant des parcours individuels singuliers. Ces traces ne sont pas accessibles à la mémoire consciente mais pourtant bien à l'œuvre, à notre insu, dans son exercice comme dans celui de la pensée. Nous y avons quelques accès dans les rêves, les lapsus, des représentations, des interdits, des comportements soutenus par d'étranges certitudes, des rituels et bien sûr dans le langage, ce que Jacques Lacan, dont Michel suivait assidûment le séminaire, a beaucoup développé avec Jacobson.

En ce sens il s'agit bien d'un système de pensée et de Freud à Lacan en passant par bien d'autres, tous se sont intéressés aux autres cultures, civilisations, comme à un enseignement pour la psychanalyse.

Freud connaissait très bien, entre autre, les travaux de Darwin, Robertson Smith, Taylor, Morgan et Frazer. Si pour certains la théorie évolutionniste prenait l'aspect d'une "enfance de l'humanité ", il ne s'en satisfaisait aucunement et ne se privait pas de faire remarquer qu'un éventuel constat n'expliquait rien et que si des humains inventaient le totem, avaient besoin de tabous, ils avaient à l'évidence des raisons psychiques sérieuses à cela. Il cherchait à saisir ce qu'il pourrait apprendre de ces autres cultures, ce qu'en retour leurs inventions pouvaient nous apprendre sur la névrose. Citant Frazer, il rappelle que "tel un caméléon, le chercheur sincère devrait changer ses couleurs pour s'adapter aux changements de couleur du sol qu'il foule ".

De même dans un très grand nombre d'articles, il ne cessera de signaler que la théorie psychanalytique devrait servir à appréhender les fondations des systèmes sociaux, quels qu'ils soient, aussi bien que leurs symptômes.

Lacan lui, autre lieu, autre époque, était très lié à Leiris, à Griaule et en 1954, dans son premier séminaire ${ }^{3}$, faisait référence à Griaule et son travail sur la cosmologie Dogon à paraître : 
"Vous allez voir bientôt dans un livre qui va paraître, qu'alors que nous ne nous servons que d'une clef très réduite celle de l'Edipe, il y en a d'autres qui ont un trousseau à leur disposition ${ }^{\prime} . .$.

"Ils en savent beaucoup plus que nous, et nous voyons que le complexe d'Edipe n'est pour eux qu'une mince petite rigolade. C'est un tout petit détail dans un mythe immense, d'une richesse et d'une complexité auprès de quoi le complexe d'Edipe ne paraît qu'une édition tellement abrégée qu'en fin de compte elle n'est pas toujours utilisable."

Jacobson, quant à lui, faisait un pont entre les descriptions de Frazer dans Le Rameau d'Or ${ }^{4}$ et les mécanismes du rêve de déplacement, la condensation et la symbolisation décrits par Freud : avec le déplacement, une chose en représente une autre. Avec la condensation, les personnages et les objets vus en rêve sont composites, un élément du rêve signifie plusieurs idées latentes à la fois et condense plusieurs éléments en un seul. Avec la symbolisation, les objets, personnes et situations apparaissent sous forme de représentations aptes à les figurer de façon analogique, et ceci jusqu'au jeu de mot.

Au rappel de ces mécanismes qui sont utilisés parce que nous appelons l'inconscient, on entend bien leur parenté avec les mythes comme avec les rituels si complexes à décrypter, qui ne sont pas des rêves, et structurent le système de pensée d'une organisation sociale.

Certains se sont servis de la psychanalyse comme d'un divan de Procuste pour évaluer les différents systèmes de société à l'aune de la lorgnette occidentale.

D'autres encore ont voulu en tirer une psychanalyse appliquée à l'usage des migrants par exemple...

Il s'agit de pervertissements, dommageables pour ce système de pensée ouvert qu'est la psychanalyse, mais qui après tout appartient à leurs auteurs. Malheureusement il en sera toujours ainsi, on le voit actuellement avec la nouvelle clef comportementale qui de la richesse de l'origine liée à l'antipsychiatrie avec Bateson, est en passe de devenir un modèle d'explication appauvri, univoque, des sociétés humaines comme des individus. Toute la richesse créative dont est capable l'humain est ainsi abrasée.

${ }^{4}$ Frazer, 1911-1915. 
Tous ces réductionnismes, aboutissant à écraser la subtile élaboration d'une société, d'une culture, d'une civilisation par le piétinement d'éléphant d'une autre, Michel Cartry les avait en horreur, et c'est peu dire.

C'était la confrontation de ce que la sophistication des systèmes de pensée différents pouvait apporter de compréhension et d'enrichissement mutuels qu'il mettait minutieusement à l'ouvrage.

A ce titre il avait organisé dans son laboratoire un groupe passionnant qui a travaillé environ trois ans, réunissant des ethnologues et deux psychanalystes, Michel Guibal et moi-même autour de l'énigmatique question de «l'objet fétiche ».

On trouve relativement peu de références directes à la psychanalyse dans ses écrits publiés. Par contre de ses recoupements de pensée, il nourrissait explicitement ses séminaires. Très fin connaisseur de Freud, Lacan mais aussi de Reik et ses écrits sur les rituels, linguiste passionné, ce qui l'intéressait, lui-même contenant de ce système de pensée qui était le sien, c'était le surgissement de la différence de traitement des affaires de l'inconscient pour et par les individus et les organisations sociales.

"Ah, mais alors eux, ils s'y prennent comme ça!" pouvait-il s'exclamer au sortir d'une exigeante recherche.

Il ne s'agit pas de mieux ou moins bien, de juste ou de faux, d'un côté ou de l'autre. C'était pour lui totalement jubilatoire de découvrir ces inventions dont l'humain est capable, et de façon souvent très sophistiquée, pour se tenir au plus près de ce qui lui échappe dans sa vie propre comme dans la mise en place de l'organisation sociale dans laquelle il est inscrit.

Dans nos sociétés, ce qui de l'inconscient est traité par la psychanalyse, pour tenter de résoudre une problématique, se fait dans la singularité d'un cabinet d'analyste.

Chez les Gourmantché, avec lesquels Michel Cartry travaillait, c'est majoritairement à travers des organisations collectives que la société traite de la problématique 
" cachée " pourrait-on dire de l'humain. Et pourtant cela n'exclut en rien les traitements individuels.

Il a abondamment montré, illustré la complexité de la présence dans tous les rituels dits de passage de prières dont une grande partie des paroles est reprise à l'identique, par fragments, d'un rituel à l'autre. Ainsi, à l'intérieur du traitement de tous chacun est renvoyé à son histoire personnelle de la naissance, reprise dans l'initiation, et reprises l'une et l'autre encore dans les funérailles. À l'intérieur d'un corpus qui se modifie à chaque occasion on retrouve des enclaves strictement identiques à travers lesquelles l'initié est un être naissant, sans métaphore, ramené au bébé naissant dans les mêmes termes, comme il en sera lors des funérailles, entrecoupées de séquences de naissance comme d'initiation et dont les récitants peuvent être des femmes qui parlent au masculin, rappel de la bisexualité originaire et fondatrice de l'humain.

Le traitement singulier des problématiques individuelles existe au même titre dans les séances de divination.

Là les questions préalables posées par le géomancien pour construire le tableau divinatoire recherchent tout ce qui a pu aller de travers dans une vie, particulièrement dans les rapports généalogiques ou les prescriptions tenteront de remettre de l'ordre. Prenons l'exemple des mauvais morts. Ceux pour lesquels on n'a pas fait ce qu'il fallait pour les funérailles, ou dont on s'est exonéré de devoirs de leur vivant. Ils errent et ne trouvent pas la paix dit-on là, mais c'est celui qui consulte pour qui les choses vont mal, enfant malade, chasseur qui n'attrape rien, maladie, stérilité.

Ici nous les connaissons aussi ces mauvais morts, ceux dont on ne parle jamais, les secrets qui les entourent, ceux qui étaient trop aimés ou trop haïs, ceux qui sont morts trop tôt avec le cortège de symptômes très divers qui les accompagnent et ne permettent pas de les rendre à leur sépulture en leur attribuant les objets qui sont les leurs et dont on se retrouve encombrés.

Le grand ami géomancien de Michel dans ce village du Burkina où il avait sa case, Iripaghba, était venu en France. 
Outre le fait qu'il m'avait un jour beaucoup fait rire en comparant le camion de livraison arrêté au milieu de la rue avec la vache qui quoi qu'on fasse ne dégagerait le chemin que quand elle en aurait décidé, nous avions beaucoup parlé de nos manières de faire respectives. Un jour il me dit : "si j'étais né ici, je croirais comme toi les choses d'ici, mais finalement on fait le même travail toi et moi..."

Mais en ce qui me concerne, je trouvais qu'il me manquait par rapport à lui cette immense perspicacité quant à ce qu'on pouvait lire de l'histoire de quelqu'un sur la structure et l'expression d'un visage, comme sur la subtilité des informations données par la manière d'habiter son corps. 


\section{Références bibliographiques}

Fazer, J. G.

1910 Totemism and exogamy, Londres, Macmillan \& co., reproduit dans Totem und Tabu \{1913\}, trad. fr. par Samuel Jankélévitch, Totem et tabou \{1924\}, Paris, Payot, 2001, p. 83.

1984 Le Rameau d'or, [The Golden Bough, 1911-1915], édition fr. par Nicole Belmont et Michel Izard, Paris, Rohert Laffont, coll. "Bouquins ", 12 volumes.

Lacan, J.

1991 Le Séminaire, Livre I, Les écrits techniques de Freud, (séminaire du 24 février 1954), Paris, Seuil, coll. (Le Champ Freudien), p. 101.

Mallarmé, S.

1945 "Variations sur un sujet. Crise de vers ", Euures complètes, in G. Jean Aubry et Henry Mondor (dir.), Paris, Gallimard, (Pléiade Bibliothèque), p. 363. 